PAPERS

\title{
Liver disease in brucellosis. A clinical and pathological study of $\mathbf{4 0}$ cases
}

\author{
F. Cervantes \\ M.D. \\ J. CARBOnell \\ M.D.
}

M. BRuguera M.D.

L. FORCE M.D.

\author{
S. WeBB \\ M.D. \\ Department of Internal Medicine and Liver Unit, Hospital Clínico y Provincial, \\ University of Barcelona, Spain
}

\begin{abstract}
Summary
Among 82 patients with brucellosis, physical and/or biochemical abnormalities suggesting liver disease were found in $\mathbf{4 0}$ cases. A soft and tender liver enlargement was present in $65 \%$ of them, and the spleen was palpable in $52 \%$. The most frequent biochemical abnormalities were a slight increase of serum transaminases and alkaline phosphatase. Liver biopsy showed a non-specific reactive hepatitis in $90 \%$ of patients, and minimal changes in the remaining $10 \%$. Non-caseating granulomas were present in 28 patients, always associated with reactive hepatitis. No differences were found when comparing clinical and biochemical features in patients with and without granulomas. However, statistically significant differences were obtained when the duration of the process was related to the type of alteration found in the liver biopsy; the finding of granulomas was practically constant when the duration of the disease before liver biopsy was under 100 days, but was infrequent after this time.
\end{abstract}

\section{Introduction}

Brucellosis is frequently observed in Mediterranean countries where it is, in some places, endemic, and should be considered in the diagnosis of longlasting fever of unknown origin (Pedro-Pons, 1977). The brucella organism's predilection for organs rich in reticuloendothelial cells (spleen, liver, bone marrow, lymph-nodes) and its intracellular location are responsible for the chronicity of the disease, which can last for months or years (Bothwell, 1963; Spink, 1964; Robbins, 1968). Hepatic involvement has been established since the first descriptions of the disease. In 1937 von Albertini and Lieberherr

Requests for reprints: Dr F. Cervantes, Clínica Médica C, Hospital Clínico y Provincial, Barcelona, Spain. described for the first time the presence of granu- $\vec{\sigma}$ lomas in the liver of patients with brucellosis. Sub- 울 sequent reports have demonstrated that liver changes, although usually subclinical, are fairly $\subseteq$ constant in brucellosis (Pedro-Pons, Bacardi and $\vec{\Phi}$ Alvarez, 1945; Spink et al., 1949; Joske and Finch $\vec{\bullet}$ 1955; Barrett and Rickards, 1959; Vivancos et alo : 1973).

The aim of the present report is to review the clinical, biochemical and histological features asso $=-2$ ciated with hepatic involvement in a series of 40 patients with brucellosis.

\section{Material and methods}

Forty patients in whom a liver biopsy has been carried out because of the presence of clinical or biochemical alterations suggesting liver disease, are included in this study. They were collected from a total series of 82 patients with brucellosis admitted to the Department of Medicine of the Hospital Clínico y Provincial over a 5-year period from 1974 until 1979.

The diagnosis of brucellosis was based on serological data. A serum agglutination test was positive $\bigcirc$ (a titre higher than $1: 160$ ) in $90 \%$ of the cases and $\supset$ high titres of anti-human globulin (Coombs') test were found in those with a negative serum agglutin- $N$ ation test. Blood cultures in Castaneda medium were positive for Brucella melitensis in 7 cases $(17.5 \%)$.

The diagnosis of brucellosis preceded the liver $\underset{2}{2}$ biopsy in 33 cases $(82.5 \%)$. In 7 cases the finding of 0 hepatic granulomas lead to the suspicion of a $\mathbb{\Phi}$ brucella infection, and later serological studies con- ? firmed the diagnosis.

Informed consent for liver biopsy was obtained in all patients. 


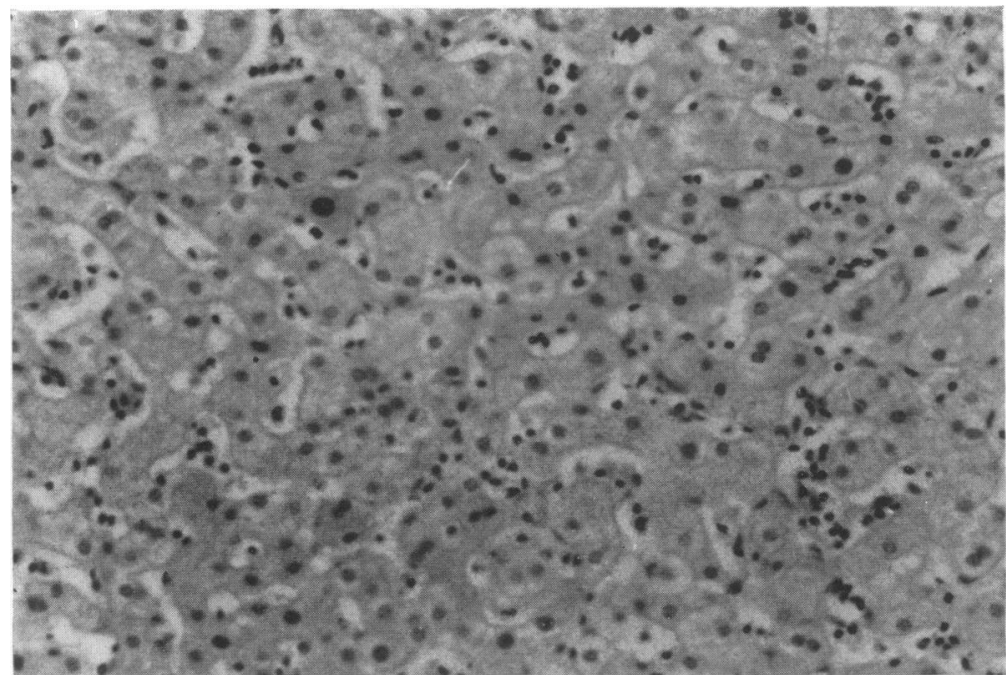

FIG. 1. Increased number and size of sinusoidal lining cells with an area of focal necrosis $(H E, \times 110)$.

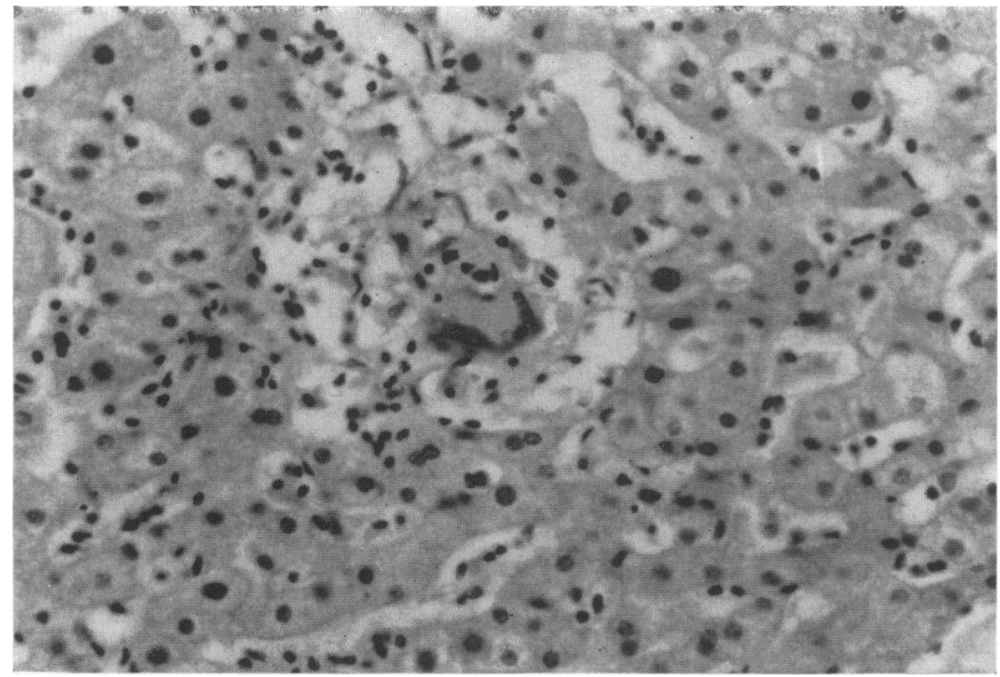

FIG. 2. Small histiocytic granuloma with a multinucleated cell $(\mathrm{HE}, \times 160)$.

The charts of the patients included in the study were reviewed for the presence of clinical data suggesting hepatic involvement and of abnormal liver function tests. A haematoxylin and eosin, a trichrome stain and a reticulin preparation of each liver biopsy specimen were reviewed by one of us (MB).

The data were statistically analysed by the $\chi^{2}$ test. A $P<0.05$ was considered to have statistical significance.

\section{Results}

Age and sex

The patients' ages ranged between 13 and 69 years, with an average of $41 \cdot 7$ years. The maximal incidence of the disease was observed in the 20-30 and $40-50$ age groups. There were slightly more males $(55 \%)$ than females.

\section{Clinical features of hepatic disease}

The liver was enlarged in 26 patients $(65 \%)$ and 


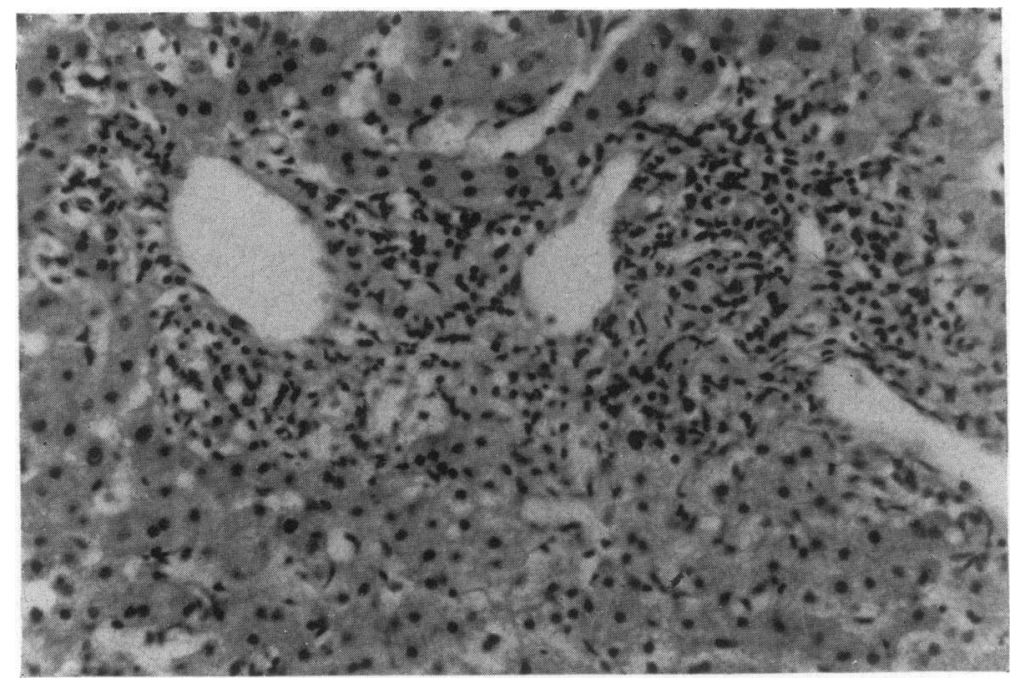

FIG. 3. Enlarged portal tract with increased cellular content. The limiting plate is not disrupted and the hepatic cords are normal $(\mathrm{HE}, \times 110)$.

the spleen in $21(52.5 \%)$. In half the cases with hepatomegaly the liver was only moderately enlarged (less than $4 \mathrm{~cm}$ below the costal margin), soft and not or only slightly tender when palpated. In the other half the liver edge was felt $4-10 \mathrm{~cm}$ below the costal margin. Among the latter, four patients were chronic alcoholics, one had congestive heart failure and one had cirrhosis.

Cutaneous jaundice was only present in two cases, and scleral icterus in one. In two cases stigmata of chronic liver disease were found on physical examination.

\section{Liver function tests}

Liver function tests were impaired in 31 patients (77.5\%). The abnormalities consisted basically of an increase of alkaline phosphatase, present in 26 patients $(65 \%)$, and an increased serum transaminase activity, present in $24(60 \%)$. In 18 patients $(45 \%)$ both alterations were simultaneously present. The average value ( \pm s.d.) for serum glutamic oxaloacetic transaminase (SGOT) was $70.4 \quad \mathrm{mu} / \mathrm{ml} \pm 48 \cdot 7$ (normal $<40 \mathrm{mu} . / \mathrm{ml}$ ), for serum glutamic pyruvic transaminase (SGPT) $61.5 \mathrm{mu} . / \mathrm{ml} \pm 44.3$ (normal $<40 \mathrm{mu} . / \mathrm{ml}$ ) and for alkaline phosphatase $158 \cdot 3 \mathrm{i}$.u./ $1 \pm 100 \cdot 7$ (normal $<90$ i.u./l). Serum bilirubin was increased in the three jaundiced patients.

\section{Liver biopsy}

All liver specimens showed morphological alterations. The most consistent change was an increase in the number and size of the sinusoidal lining cells, associated with a marked increase in portal cellular content and some focal lobular necrosis (Fig. 1). This was the case in 35 patients $(90 \%)$, and was interpreted as non-specific reactive hepatitis. In 28 patien $\mathscr{\mathscr { Q }}$ (70\%), single or multiple granulomas were found i i addition to these mesenchymal reactions. The majority had a lobular location and were compose of lymphocytes, histiocytes, epithelioid cells and occasional multinucleated cells (Fig. 2). No central caseation was observed, and special stains for acid $\stackrel{\varnothing}{\varnothing}$ fast bacilli, fungi and bacteria were negative. In four cases $(10 \%)$ only minimal changes were found, such as small histiocytic nodules in the parenchyma and a mild increase of round cells in the portal tracts (Fig. 3). In one case the biopsy showed micronodular hepatic cirrhosis associated with the presence of granulomas. Fatty changes were present in four patients, two of whom were diabetics.

No differences were found regarding clinical manifestations, particularly liver enlargement or abnormal liver function test, between the patients whose liver biopsy contained granulomas and those who did not. However, significant differences in the duration of the disease until the moment the biopsy was carried out were found: $52 \pm 36$ days in the patients $\tilde{\sigma}$ with granulomas and $137 \pm 219$ days in those $N$ without granulomas $(P<0.05)$. In only 3 of the 28 N patients with granulomas was the duration of clinical $\omega$ manifestations longer than 100 days, while this occurred in 6 of the 12 patients without granulomas.

\section{Outcome}

After appropriate treatment with streptomycin, tetracycline and sulphonamides the clinical and 
biochemical manifestations of liver disease disappeared in all cases within a period ranging from 15 days to 6 months.

\section{Discussion}

Involvement of the liver in brucellosis has been demonstrated in clinical and experimental studies for many years (von Albertini and Lieberherr, 1937; Barrett and Rickards, 1959; Braude, 1959). Fabyan described in 1912 the pathological changes in experimental brucellosis in guinea-pigs, showing that lesions were more prominent in tissues rich in reticuloendothelial cells. Braude (1959) studied the changes occurring in mice after peritoneal inoculation of B. abortus. After $3 \mathrm{hr}$ organisms were found inside polymorphonuclear leucocytes in liver sinusoids and Kupffer cells; after $24 \mathrm{hr}$ the number of polymorphonuclear leucocytes had decreased in the liver, and the number of organisms had increased in Kupffer cells. Later, granulomas developed, formed almost exclusively by epithelioid cells. After 6 months the granulomas began to reabsorb and after one year they had disappeared without sequelae. From the presence of this non-suppurative granuloma, Braude inferred an effective defence mechanism against the organism. The larger the focal collection of phagocytes, the less numerous were the intracellular organisms. The course of the disease was particularly favourable in those cases in which there was a clear predominance of mononuclear cells in the granulomas, whereas the persistence of polymorphonuclear leucocytes with formation of abscesses was associated with an unfavourable course.

Hepatic granulomas with or without necrosis, indistinguishable from tuberculous granulomas, were first observed in human brucellosis by von Albertini and Lieberheer (1937). Later several reports describing the pathology of the liver in brucellosis appeared (Pedro-Pons et al., 1945; Spink et al., 1949; Joske and Finch, 1955; Barrett and Rickards, 1959; Vivancos et al., 1973). Histological hepatic changes were observed both in patients with and without clinical or biochemical signs of liver disease, indicating that liver involvement is very frequent in brucellosis.

Half the patients with brucellosis seen in our hospital had some clinical or biochemical evidence of liver disease. The most frequent clinical feature was an enlarged liver, present in $65 \%$ of patients with liver disease, whereas a palpable spleen was detected in $52 \%$, a frequency similar to that observed in other studies (Joske and Finch, 1955; Barrett and Rickards, 1959; Vivancos et al., 1973; Santillana et al., 1976). The liver enlargement was usually moderate and soft. Where the liver was larger and firmer, there was often an associated pathology, which possibly accounted for this finding. Icterus was seldom found.

Changes in liver function tests were non-specific. They consisted generally of a moderate increase of alkaline phosphatase and serum transaminase activity. These were occasionally the most prominent manifestation of the disease, which was suspected after the observation of granulomas associated with changes of non-specific reactive hepatitis in the liver biopsy, and occurred in 7 of our patients.

In our series the most common histological abnormalities were a pattern of non-specific reactive hepatitis, which was present in the majority of cases, and granulomas, which were found in $70 \%$ of the liver biopsies. In most series the presence of hepatic granulomas ranges between 50 and $100 \%$ of cases (Spink et al., 1949; Joske and Finch, 1955; Barrett and Rickards, 1959; Vivancos et al., 1973). Granulomas seen in brucellosis may be indistinguishable from those found in other diseases, such as sarcoidosis, tuberculosis and histoplasmosis, but they can be smaller and less clearly defined than those seen in these diseases. Thus, it is our feeling that the observation in a liver biopsy specimen of small granulomas associated with portal and interstitial inflammatory infiltrates in the clinical context of fever of unknown origin should suggest the diagnosis of brucellosis and lead to the relevant serological examinations, particularly in those areas where brucellosis is endemic.

It has recently been stated that hepatic granulomas are present in brucellosis due to $B$. abortus and absent in cases due to $B$. melitensis (Young, 1979). In a small series of 7 patients with bloodculture-proven brucellosis, Young found noncaseating granulomas in the liver of two patients with infection due to $B$. abortus while in the remaining patients infected by $B$. melitensis no granulomas were observed. The present study does not support this finding, for granulomas were present in the liver biopsies from 4 of the 7 patients with bloodculture positive for $B$. melitensis. Furthermore, Masana et al., (1980) found granulomas in 9 of 31 patients with $B$. melitensis infection in whom a liver biopsy was done.

Especially interesting is the fact that in our series granulomas were rarely found beyond 100 days of disease. The presence of liver granulomas in patients with a short history has led to the belief that granulomas are encountered in active or relatively acute brucellosis (Joske and Finch, 1955), while portal tract infiltration and fibrosis occurs in all stages but mainly in long standing brucellosis. Our findings support this hypothesis.

In the only case where the liver biopsy disclosed cirrhosis, granulomas were simultaneously present; chronic liver disease was known to exist before the 
brief febrile illness which caused the patient's admission to the hospital, an aetiological relationship between the infection and the development of cirrhosis thus being unlikely. Although brucellosis of long duration or resistant to treatment has been implicated in the production of cirrhosis (Spink et al., 1949; McCullough and Eisele, 1951), it seems unlikely that a miliary type lesion like the one seen in brucellosis could produce enough fibrosis to justify the term cirrhosis. Furthermore, cirrhosis has never been produced by experimental infections (Sherlock, 1975). In all our patients evidence of liver disease disappeared shortly after treatment began.

\section{References}

Albertini, von A. \& LieberherR, W. (1937) Beitrage zur pathologischen Anatomie der Febris Undulars Bang. Frankfurter Zeitschrift für Pathologie, 51, 69.

BarretT, G.M. \& Rickards, A.G. (1959) Chronic brucellosis. Quarterly Journal of Medicine, 22, 23.

Bothwell, P.W. (1963) Brucellosis. The Practitioner, 191, 577.

Braude, A.I. (1959). The evolution and significance of the hepatic granuloma in experimental brucellosis. Journal of Clinical Investigation, 29, 799.

FABYAN, M. (1912) A contribution to the pathogenesis of B. Abortus; Bang II, Journal of Medical Research, 26, 441.
Joske, R.A. \& FINCH, E.S. (1955) Hepatic changes in human brucellosis. Medical Journal of Australia, 1, 266.

Masana, L., Bernardo, L., Bacardi, R. \& Guardia, J. $\stackrel{\mathbb{Q}}{\varrho}$ (1980) Brucella hepatitis. Annals of Internal Medicine, 92, $\subseteq$ 709.

McCullough, N.B. \& Eisele, C.N. (1951) Brucella hep- क्⿹ atitis leading to cirrhosis of the liver. Archives of Internal 0 Medicine, 58, 793.

Pedro-Pons, A. (1977) Patología y Clinica Médica. Salvat Editores, Barcelona.

Pedro-Pons, A., Bacardi, R. \& Alvarez, R. (1945) Hígado $₫$ melitocócico. Medicina Clinica (Barcelona), 5, 16.

RobBins, S.L. (1968) Pathology. W. B. Saunders, Philadelphia.

Santillana, T., Castañeda, E., Fraga, I. \& Ruiz, A. $\overrightarrow{0}$ (1976) Brucelosis: consideraciones clínicas y terapeúticas. $\vec{\longrightarrow}$ Revisión de 50 casos. Revista clinica española, 141, 131.

SHERLOCK, S. (1975) Diseases of the Liver and Biliary System. 5th edn Blackwell Scientific Publications, Oxford.

Spink, W.W., Hoffbauer, F.W., Walker, W.W. \& Green, 3 R.A. (1949) Histopathology of the liver in human brucel- ir losis. Journal of Laboratory and Clinical Medicine, 34, 40.

SPINK, W.W. (1964) Host-parasite relationship in human brucellosis with prolonged illness due to suppuration of the liver and spleen. American Journal of the Medical $\omega$ Sciences, 35, 129.

Vivancos, J., Bruguera, M., Cabrer, B., Diaz, F. \& 0 Salamero, P. (1973) Lesiones hepáticas en la brucelosis. Revista clinica española, 131, 119.

Young, E.J. (1979) Brucella melitensis: The absence of granulomas. Annals of Internal Medicine, 91, 414. 